http://dx.doi.org/10.18778/2196-8403.2018.02

KATARZYNA WÓJCIK

\title{
Der Blick auf die Münchener Räterepublik im Revolutionstagebuch 1919 von Victor Klemperer
}

Victor Klemperer beschreibt in seinem Revolutionstagebuch 1919 die Ereignisse der Münchener Räterepublik als Tragikomödie - eine Mischung aus Lächerlichkeit, Verbrechen sowie Bitterkeit - und übt zugleich Kritik an dem konservativen Münchener Bürgertum. In anschaulichen Momentaufnahmen aus der belagerten Stadt schildert er das Scheitern der Revolution 1918/1919, einen der entscheidenden Wendepunkte der deutschen Geschichte.

A look at the Bavarian Soviet Republic in Victor Klemperer's Revolutionstagebuch 1919 In his book Revolutionstagebuch 1919, Victor Klemperer presents events taking place during the time of the Bavarian Soviet Republic as a tragicomedy - a mixture of ridiculousness, bitterness and crime, while fiercely criticising the mentality of the conservative bourgeoisie of Munich. In his vivid descriptions of successive events as they unfold in the besieged city, the author depicts the defeat of the 1918-1919 Revolution - one of the most decisive moments in German history.

Spojrzenie na Bawarską Republikę Rad w Revolutionstagebuch 1919 Victora Klemperera Victor Klemperer opisuje w swoim pamiętniku Revolutionstagebuch 1919 wydarzenia w Bawarskiej Republice Rad jako tragikomedię - mieszankę śmieszności, zbrodni i goryczy, krytykując jednocześnie konserwatywne monachijskie mieszczaństwo. W obrazowych wycinkach rzeczywistości z oblężonego miasta ukazuje klęskę rewolucji 1918/1919 roku, jednego $\mathrm{z}$ decydujących momentów w historii Niemiec.

Die Revolution vom November 1918 - ein folgenschwerer Wendepunkt in der deutschen Geschichte - ist Thema mehrerer geschichtswissenschaftlicher (vgl. BIEBER 1991; KLUGE 1985; BISCHOFF 1977; MEYER 1977) und literaturwissenschaftlicher (vgl. CHOŁUJ 1991; KITTSTEIN / ZELLER 2009; KREILER 1978) 
Studien und Beiträge. Die Verwirrung der Novembertage 1918 beschrieben u. a. der Mitbegründer der Weimarer Republik, Partei- und Fraktionsvorsitzende der SPD, Außenminister und zweimalige Reichskanzler Hermann Müller (vgl. MÜLlER 1929), der Schriftsteller Friedrich Burschell (vgl. BuRSCHELl 1962) und der Schriftsteller Klaus Mann (vgl. MANN 1967), damals ein junger Schüler. In die revolutionären Ereignisse in Deutschland waren Politiker, Schriftsteller sowie Künstler wie Kurt Eisner, Gustav Landauer, Ernst Toller, Erich Mühsam, Oskar Maria Graf, Max Weber, Georg Kaiser, Alfred Wolfenstein, Heinrich Mann, Bruno Frank, Friedrich Burschell, Ret Marut verwickelt (vgl. WEILLER 1994:167). Unter den Augenzeugen der Münchner Räterepublik lassen sich zwei Gruppen unterscheiden: Beteiligte und Beobachter. Der junge Journalist Victor Klemperer gehörte zweifellos zu der zweiten Gruppe. Klemperer, für den die Münchner Revolutionäre lächerliche Schwärmer waren (KLEMPERER 2015a:54f.) und der Kurt Eisner für einen „Feuilletonisten“ hielt (KLEMPERER 2015a:52), ließ sich selbst damals als entschiedener Gegner der Revolution erkennen. Die Schriftsteller, die Regierungsposten übernahmen, wie Ernst Toller (WEIDERMANN 2017:273f., 282f.), Gustav Landauer (WEIDERMANN 2017:253f.) und Erich Mühsam (WEIDERMANN 2017:280f.), die zuerst die Aufbruchsstimmung der Münchener Revolution beherrschte, fielen ihr selbst zum Opfer. Es gab auch solche wie Rainer Maria Rilke (HÖLLER 2018) und Oskar Maria Graf (HÖLLER 2018), bei denen der euphorischen Begeisterung rasch Ernüchterung folgte. Kaum weniger von den Wirren der Münchener Revolution beeindruckt zeigte sich der namhafte Thomas Mann (WEIDERMANN 2017:259-262), der damals von den Extremen der Ereignisse hin- und hergerissen war.

Probleme mit der Schilderung und Rezeption der Münchener Räterepublik aus historischer Perspektive lassen sich nicht auf die Weichenstellung der Jahre 1918/1919 zurückführen. Nach Sebastian Haffner, einem der bekanntesten Chronisten der deutschen Geschichte des 20. Jahrhunderts, vollzog sich die Revolution in Bayern anders als im übrigen Deutschland. Es gab hier seiner Meinung nach kein Legitimationsdefizit. Die erste friedliche Revolutionsetappe hatte einen Anführer, Kurt Eisner, dank dem zahlreiche Reformen in Angriff genommen wurden (HAFFNER 1991:169). Aus seinen Ausführungen geht hervor, dass die Münchener Ereignisse von 1919 „kein Schandfleck der nationalen Geschichte" seien, sondern ,eine Ruhmestat", die Bayern nach dem Elend des Ersten Weltkriegs und im Angesicht des politischen „Verrats“ vollbrachten (HAFFNER 1991:169, 213). 
In seinem vor kurzem bekannt gewordenen Tagebuch Man möchte immer weinen und lachen in einem. Revolutionstagebuch 1919, das in seinem in der Sächsischen Landes- und Universitätsbibliothek in Dresden aufbewahrten Nachlass entdeckt wurde (vgl. REICHWEIN 2015), schildert Victor Klemperer die historischen Ereignisse und ihre Hintergründe, Schauplätze und Personen sowie deren Handlungen zur bewegten Zeit der Münchener Räterepublik (vgl. SCHMALZL 2017).

Im November 1915 meldete sich Victor Klemperer als Kriegsfreiwilliger und diente bis zum März 1916 an der Front (JACOBS 2010:73f.). Die letzten Jahre des Ersten Weltkriegs verbrachte er zunächst als Zensor im Buchprüfungsamt der Presseabteilung des Militärgouvernements Litauen in Kaunas und dann in Leipzig, wo zu Kriegsende auch seine Frau Eva lebte (JACOBS 2010:79-84). Mitte Dezember 1918 reiste Klemperer nach München „für möglichst wenige Tage“ (KLEMPERER 2015a:27). Er bemühte sich um seine ordnungsgemäße Entlassung aus dem Militärdienst und wollte seine Universitätstätigkeit als Privatdozent für moderne französische Literatur an der Ludwig-MaximiliansUniversität wieder aufnehmen (vgl. KLEMPERER 2015a:27f.). Anfang des Jahres 1919 bezogen die Klemperers eine Wohnung in München (vgl. KLEMPERER 2015a:32f.). Eva Klemperer wollte in München ihr Orgelstudium fortsetzen (vgl. KLEMPERER 2015a:32). Paul Harms, der Leitartikler der nationalkonservativen Leipziger Neuesten Nachrichten, ermutigte den Privatdozenten Victor Klemperer, als Korrespondent Berichte aus München zu schreiben, was dieser mit einer gewissen Zurückhaltung betrachtete: „Wir lachten, und dann wurde von anderem gesprochen. Im Augenblick legte ich Harms' Worten keine Bedeutung bei. Die Politik versank wieder einmal für mich. Jetzt, wo ich in sechs Wochen zum erstenmal auf ein wirkliches deutsches Universitätskatheder sollte“ (KLEMPERER 2015a:55f.). Die Revolution war für Klemperer zunächst zwar nur ein Nebenkriegsschauplatz, aber im Alltag schien sie ihn zu bedrängen: „Und die Revolution sollte mich nicht stören. Ich wollte arbeiten, nichts als arbeiten: [...] aber wirklich ausschalten ließ sich die Revolution doch nicht, sie war immer da, vom Morgen bis zum Abend“ (KLEMPERER 2015a:21f.). Während der Arbeit an der LTI 1942 schrieb er im Rückblick auf seine journalistischen Berichte aus der Münchener Räterepublik (KLEMPERER 2015c:44, 68), er habe damals seine ,alte Sehnsucht nach der politischen Journalistik ein für allemal abreagiert" (KLEMPERER 2015a:66.). Die innere Verflechtung der beiden Rollen - einerseits der eines Wissenschaftlers, der sich um die Fortsetzung seiner akademischen Laufbahn bemühte, und andererseits der eines hellwachen Journalisten - nahm ihren Anfang am siebten Februar, noch bevor er seinen ersten Artikel schrieb (vgl. KLEMPERER 2015a:66). Mit seinem Pseudonym „A.B.- 
Mitarbeiter" (=Antibavaricus) wollte er erst seinen potenziellen Leserinnen und Lesern zu verstehen geben, dass er die Münchener Revolution nicht ernst nimmt (KLEMPERER 2015a:30): ,[E]s war eine Gaudi, ein politischer Fasching“. „Gaudi“ (KLEMPERER 2015a:15, 30, 141, 146) und „Fasanenjagd“ (KLEMPERER 2015a:132, 145, 175) gehören eben zu den von Klemperer meistbenutzten Bezeichnungen für die erste Phase der Münchener Revolution.

Die Doppelstruktur des Textes basiert auf zwei Textsorten - einerseits private Tagebuchnotizen und andererseits journalistische Berichte, die er als Korrespondent der Leipziger Neuesten Nachrichten lieferte. Wegen der gesperrten Postwege gelangten nicht alle Berichte Klemperers aus dem München der Revolutionszeit an ihr Ziel. Schätzungsweise konnte nur jeder dritte von den 15 verfassten Berichten veröffentlicht werden (vgl. KLEMPERER 2015a:65). 1942 überarbeitete Klemperer seine eigenen Aufzeichnungen über die Münchener Räterepublik von 1919 nochmals. Die Doppelstruktur des Revolutionstagebuches, die durch zwei verschiedene Aufzeichnungsmodi entstand, wurde um die Kennzeichnung der beiden Zeitebenen (1919 und 1942) ergänzt. Die zweispaltig gesetzten Zeitberichte des Revolutionstagebuches 1919 entstanden in der Zeit der Münchener Räterepublik zwischen Februar 1919 und Januar 1920 und wurden erst in den Leipziger Neuesten Nachrichten als Berichtserie veröffentlicht. Texte im klassischen Satzspiegel sind Teile von Klemperers Erinnerungen, die nicht in den Sammelband Curriculum vitae. Erinnerungen 1881-1918 eingegangen sind, weil sie nach Klemperers Konzept ursprünglich zu einem größeren Kapitel mit dem Untertitel Privatdozent gehören sollten (vgl. KLEMPERER 2015a:9). Es konnte im Jahre 1942 nicht mehr weitergeschrieben werden, da die Gefahr von Hausdurchsuchungen durch die Gestapo damals zu groß war. Klemperer brachte zwar viele Materialien aus der Kriegsund Revolutionszeit, die er fertigstellen wollte, in das Dresdner Judenhaus, aber er gab sie dann mit einem Exemplar des Curriculums und den übrigen nicht fertiggestellten Manuskripten Annemarie Köhler zur Aufbewahrung, mit der Bitte, falls ihm etwas zustoßen sollte, alle Schriftstücke der Dresdner Staatsbibliothek zu übergeben (vgl. JACOBS 2010:228; KLEMPERER 2015c:44). Bemerkenswert ist, dass Klemperer, der die Revolution 1919 einer niederschmetternden Kritik unterwarf, seine Einstellung nach 23 Jahren nicht änderte, obwohl in LTI seine Sympathien zweifellos auf der Seite der Kommunisten liegen (vgl. KLEMPERER 2015b:178f.). Die rückblickenden Passagen aus Klemperers Erinnerungen wurden jedoch in der Nazi-Zeit um eine tiefergehende Reflexion des verfolgten Dresdener Juden bereichert. Die Revolutions- 
zeit wurde dann im Jahre 1942 aus der biographischen und historischen Perspektive verurteilt. In den Erinnerungen nahm Klemperer die zeitgenössische Perspektive eines jungen Journalisten ein. Im nationalsozialistischen Dresden meldete sich seine Stimme des Zwangschronisten zu Wort, eben zu der Zeit, als er wegen seiner jüdischen Abstammung der deutschen Staatsangehörigkeit und ihm zustehender Bürgerrechte beraubt wurde, was letztendlich in Frustration mündete: „Man kann immer nur subjektiv deuten, nicht mehr objektiv wissen. [...] Que sais-je? Und que sais-je? Ich als Jude vom heutigen Zustand der arischen Deutschen?" (KLEMPERER 2015c:34). Der historische Kontext der Münchener Revolution erschien dem verfolgten Juden Klemperer in der Rückschau eher tragisch als lächerlich. Eins ist sicher - die sozialen Erfahrungen des Alltags 1918-1919 verflochten sich mit der politischen Wirklichkeit, die sich vor alles andere schob, zu einer Einheit. In seinen ergänzenden Kommentaren aus dem Jahre 1942 brachte er diesmal mehr das Tragische der Gegenwart ans Licht, wobei das Lächerliche an der Münchener Revolution ferner dem beträchtlichen Teil seiner Beobachtungen zufiel. Klemperer, als Preuße in Landsberg an der Warthe und dann in Berlin aufgewachsen, äußerte sich über Bayern polemisch und kritisch mit zugespitzter Ironie. Er betonte Münchens „karnevalistischen Anstrich“ (KLEMPERER 2015a:31), „bunte Fahnen und Fähnchen“ (KLEMPERER 2015a:28f.), und die Münchener Bohème sei für ihn ,eine Fremdlegion“ (KLEMPERER 2015a:15), die zur Belustigung (zur Gaudi) erst geschaffen, jetzt statt der Kunst die Politik betreibe (KLEMPERER 2015a:15). Klemperer gab das allgemein herrschende Chaos und die Atmosphäre der Stadt mit den sich an Litfaßsäulen, Maueranschlägen, Plakatmauern, Zeitungsständen zusammendrängenden Menschen in fast szenischen Schilderungen wieder:

In den folgenden Monaten bedeuteten mir diese Menschentrauben ein vertrautes und sicheres Zeichen, sie erinnerten mich dann immer an das Blasenwerfen kochender Milch, das ihrem Überschäumen um ein paar Sekunden vorausgeht; ich wußte beim Auftauchen der runden Häufchen genau, daß wir in spätestens 24 Stunden bestimmt einen Streik der Trambahner, fast sicher Generalstreik und sehr wahrscheinlich ein Feuergefecht zu erwarten hatten. (KLEMPERER 2015a:29f.)

Als Klemperer nach München fuhr, amtierte schon der ehemalige VorwärtsRedakteur und Vorsitzende des Arbeiter- und Soldatenrates Kurt Eisner als bayerischer Ministerpräsident, nachdem er führend und in Zusammenarbeit mit der USPD (Unabhängige Sozialdemokraten) am Sturz des bayerischen 
Königs Ludwig III. beteiligt war und den ,Freistaat Bayern' ausgerufen hatte. ${ }^{1}$ Die Revolutionsregierung hielt der voranschreitenden Radikalisierung der politischen Szene Bayerns nicht stand. Bei den im Januar 1919 stattfindenden bayrischen Landtagswahlen erhielt Eisners USPD nur 2,5 \% der Stimmen. Die SPD wurde mit 33\% der Stimmen zweitstärkste Kraft hinter der konservativen antisemitischen Bayerischen Volkspartei, die 35\% der Stimmen bekam (vgl. WETTE 2015:207f.). Mit der Ermordung Kurt Eisners durch den rechtsradikalen und antisemitischen Jurastudenten und beurlaubten Leutnant der Infanterie Anton Graf Arco auf Valley endete die kurze politische Episode, in der zwar keine tiefgreifenden politischen Veränderungen vorgenommen wurden ${ }^{2}$, deren Ausgang jedoch die vorherige Lächerlichkeit (in München und namentlich an der Universität war damals „fei nix z' lachen“) (KLEMPERER 2015a:84) durch Grausen ersetzte (WETTE 2015:208). Am 26. Februar 1919 fand Kurt Eisners Beerdigung statt, bei der er von hunderttausend Menschen, die am Trauerzug teilnahmen, geehrt wurde (WETTE 2015:208). Klemperer stellte mit Erstaunen fest, dass „ein zartes, winziges, gebrechliches, gebeugtes Männchen“, dem „niemand den reinen Willen absprechen konnte“ (KLEMPERER 2015a:51f.) und dem von dem ,echten' Münchener Volk bei der Wahlkampfveranstaltung zugejubelt wurde, posthum zum Märtyrer stilisiert wurde und „viel mehr Anhänger [habe], als der lebende je besessen hat" (KLEMPERER 2015a:86). Klemperer verurteilte zugleich mit scharfen Worten den tödlichen Mordanschlag auf Eisner:

Er wollte seine Hände rein halten von Geld und Blut. Er hatte immer den besten Willen, und er setzte bei anderen Menschen, mit Vorliebe bei denen im Lager der Entente, wo man den Schein der Humanität so bewundernswert gut um das brutalste Machtverlangen zu schleiern weiß - er setzte dort [...] die gleiche Seelenunschuld voraus. (KLEMPERER 2015a:86)

Er beschrieb auch die bedrohliche Stimmung in der Stadt mit der Aufforderung zum Generalstreik bis zur Besetzung von Presse und Post und der Bewaffnung der Arbeiterschaft (vgl. KLEMPERER 2015a:90-93). Die Ermordung Eisners vertiefte die politische Krise und löste Chaos und Attentate im Landtag aus

1 Die Münchener Revolution lässt sich in 3 Phasen unterteilen. Die erste Etappe vollzog sich vom 8. November 1918 mit der Ausrufung des Freistaates Bayern bis zur Ermordung Eisners am 21. Februar 1919 (vgl. GRAU 2008).

2

Die zweite Etappe der Münchener Revolution begann mit dem Tod Eisners und dauerte bis zur Ausrufung der Münchner Räterepublik (vom 21. Februar bis zum 7. April 1919) (vgl. GraU 2008). 
(vgl. KLEMPERER 2015a:83f.). Der neugewählte Ministerpräsident und Mehrheitssozialdemokrat Johannes Hoffmann, der ab dem 17. März amtierte, konnte die politische Stabilisierung in der Landeshauptstadt nicht gewährleisten. Die extreme Linke nutzte die politische Trägheit der legitimen Regierung aus. Am siebten April 1919 wurde in München durch den Zentralrat der bayerischen Republik die „Bayerische Räterepublik“ ausgerufen. Die Räterepublik musste vielen angestauten Problemen die Stirn bieten - u. a. der Lebensmittelversorgung (KLEMPERER 2015a:73, 142), dem Generalstreik (KLEMPERER 2015a:88-90, 145f.), der Schließung der Banken (KLEMPERER 2015a:114f.) sowie der eigenen Unfähigkeit (KLEMPERER 2015a:86-88, 116-118, 142-144, 153-160). Ministerpräsident Hoffmann wurde für abgesetzt erklärt und musste mit seiner Regierung nach Bamberg fliehen. In dem misslungenen ,Palmsonntagsputsch“ vom 13. April gelang es den regierungstreuen Putschisten nicht, die Räterepublik abzusetzen. Die führenden KPD-Mitglieder - Eugen Leviné, Max Levien, und Rudolf Egelhofer, der Münchener Stadtkommandant - nutzten die Situation und riefen die kommunistische Räterepublik aus (vgl. SCHMALZL 2017). Zu den spektakulärsten Maßnahmen, die in der radikalsten und brutalsten Phase ergriffen wurden, gehörten die Anwerbung der Roten Armee sowie die Entwaffnung des Bürgertums. Wegen der Umzingelung der Stadt und der Verstärkung der Freikorpsverbände mit regulären, durch die Reichsregierung in Marsch gesetzten Armee-Einheiten wurde München von der überlegenen militärischen Macht der Weißen erobert, wobei jeder Widerstand mit brutaler Gewalt im Keim erstickt wurde. Im Mai 1919 marschierten die Weißen Truppen in München ein (SCHMALZL 2017). Es kam zu beispiellosen Gewalttaten (KLEMPERER 2015a:162). ${ }^{3}$

Die Revolution war für den entschiedenen Liberalen Klemperer (vgl. KLEMPERER 2015a:62): „ein Hohn auf die Demokratie. [...] Ein Hohn auf die Freiheit! (KLEMPERER 2015a:92) sowie ein „Mischmasch aus Lächerlichkeit und Verbrechen“ (KLEMPERER 2015a:169). Der unblutige Verlauf der Revolution bis zur Ermordung Eisners wurde erst durch eine beklemmende Unsicherheit ersetzt: „Die einzige Gewißheit ist der Zweifel an allem“ (KLEMPERER 2015a:86). Zwei Monate später notierte Klemperer: „Schwabing spielt Weltrevolution - es wäre zum Lachen, wenn es nicht auch zum Verzweifeln wäre, weil eben die zunehmende Verbohrtheit der eigentlichen Proletarier kein Spiel ist" (KLEMPERER

3 Die dritte Etappe der Münchener Revolution vom 7. April bis zum 1. Mai 1919 endete mit der gewaltsamen Eroberung Münchens durch Reichswehr- und Freikorpstruppen (vgl. Grau 2008). 
2015a:135). Kommunistischer Fanatismus ist an die Stelle des schwärmerischen Idealismus getreten. Klemperer schrieb in seinem Revolutionstagebuch, wie „Abenteurer“, „Schwärmer- und Bohémiennaturen mit Notwendigkeit von Stunde zu Stunde robusteren Verbrechergestalten weichen müssen" (KLEMPERER 2015a:117). Ahnungslosigkeit und Passivität schienen von allen Bevölkerungsschichten Besitz ergriffen zu haben: „München nimmt sein tragikomisches Schicksal passiv hin, auch das scheinbar herrschende Proletariat ist ganz passiv, es läßt sich da- und dorthin schieben“ (KLEMPERER 2015a:117). Mehr als je zuvor schien die Stadt ein ,überaus kriegerisches Gepräge“ (KLEMPERER 2015a:120) zu pflegen. Es wurden radikale Maßnahmen gegen die Stadtbewohner ergriffen. So hatten die Bürger Waffen abzugeben, wurden Hausdurchsuchungen nach gehamsterten Lebensmitteln durchgeführt, kam es zur Beschlagnahmung des Universitätseigentums (vgl. KLEMPERER 2015a:120-123) und Einführung des Presseverbots, wurden bürgerliche Geiseln genommen (vgl. KLEMPERER 2015a:133). Die Apathie der Münchener Bürger fand Klemperer symptomatisch wie symbolisch - etwa „die stoische Ruhe, mit der das Münchener Bürgerehepaar stundenlang im Fenster liegt, so unbewegt, als gehöre es zur Architektur des Hauses. Immer wieder: das eigentliche München sieht dem Revolutionsspiel fremder närrischer Gesellen zu“ (KLEMPERER 2015a:124). Nach den blutigen Ereignissen Ende April und Anfang Mai reflektierte er kritisch die politische Lage, indem er vor Hoffmannschen ,Lösungen“ sowie Konsequenzen der politischen Unfähigkeit warnte, und das bürgerliche Engagement und die Unterstützung einer zukünftigen Regierung als unentbehrlich ansah: „Ein Mann und kein Hoffmann muß uns leiten, und Bürgerliche müssen ihm männlich zur Seite stehen“ (KLEMPERER 2015a:182). Es lässt sich eine Parallele ziehen zwischen seinen Erwägungen nach Eisners Tod, als sich Klemperer mit dem bösen Schlagwort der „,zweiten Revolution“ - „Unsicherheit" (KLEMPERER 2015a:87) - auseinanderzusetzen versuchte, und dem sorgenvollen Blick in die unabsehbare Zukunft nach den folgenschweren Tagen im April/Mai 1919: „Ein Ansatz zu neuer politischer Entwicklung nach der blutigen Katastrophe ist noch nicht recht erkennbar" (KLEMPERER 2015a:172).

Sein Talent zur szenischen Schilderung brachte er auch angesichts des Einmarsches der Reichswehrtruppen zur Anwendung, indem er schrieb, dass „neun Zehntel von München den Befreiern zujubelte [...] (KLEMPERER 2015a:168), „wie ein friedliches Volksfest“" (KLEMPERER 2015a:165), was Trauben von Zuschauerinnen und Zuschauern mit Operngläsern beobachteten (KLEMPERER 2015a:168). Nach acht Tagen, als die Jubelstimmung nach dem Einmarsch der 
Freikorps- und Reichswehreinheiten abgeklungen war, versuchte Klemperer in groben Zügen die tragischen und grässlichen Folgen der Gegenrevolution zu schildern:

[...] es herrscht keine Feststimmung in München, sondern Sorge und Bedrückung auf der einen, furchtbare Erbitterung auf der anderen Seite. Allzu wütend ist der Kampf gewesen, und der verzweifelte Widerstand der Kommunisten überstieg alle Erwartung. [...] München, um das Fünf- bis Sechsfache kleiner als Großberlin, hat mindestens ebensoviel Blut fließen sehen. (KLEMPERER 2015a:176f., 241)

$\mathrm{Zu}$ den erschütterndsten Reflexionen im Revolutionstagebuch gehören zwei Aufzeichnungen: die erste vom 2. Mai 1919 über den Geiselmord an Münchener Bürgern, die „[...] dem systematisch vertierten Pöbel überliefert [wurden]“ (KLEMPERER 2015a:163) und die zweite über die Erschießung von 21 Mitgliedern des katholischen Gesellenvereins in ihrem Vereinslokal durch Regierungstruppen wegen einer falschen Anzeige, die gegen sie - als vermeintliche Spartakisten - erstattet wurde (vgl. KLEMPERER 2015a:177, 184, 241).

Die Revolution 1918/1919 konfrontierte den zum Protestantismus konvertierten Sohn eines Rabbiners mit dem in verschiedenen Münchener Bevölkerungsschichten (im Bürgertum, bei den Mitgliedern der Bayerischen Volkspartei, bei den Universitätsprofessoren, bei den Studenten sowie den Freikorpssoldaten) aufkeimenden Antisemitismus. Judenfeindlichkeit beobachtete Klemperer in allen Phasen der Münchener Revolution. Die sogenannte Gegenrevolution missachtete ihm zufolge führende Persönlichkeiten der gescheiterten Münchener Räterepublik, unter denen es radikale Politiker und Literaten gab - Kurt Eisner, Gustav Landauer, Max Levien, Eugen Leviné, Erich Mühsam, Ernst Toller, die pauschal öffentlich als „Saujuden“ bezeichnet wurden (WETTE 2015:213). ${ }^{4}$ Die haltlosen Beschuldigungen gegen Juden wurden damals in München und in ganz Deutschland, aber auch in den folgenden Jahren ein politisches und gesellschaftliches, signum temporis'. Klemperer musste in dieser Zeit den gegen Juden gerichteten Vorurteilen die Stirn bieten. Er war jedoch Deutscher aus Überzeugung (vgl. KLEMPERER 2015a:186) und behauptete, im Allgemeinen mit seiner ,akademischen und bürgerlichen Umgebung“ (KLEMPERER 2015a:96) in Eintracht zu leben. Die von der damaligen bürgerlichen Propaganda geprägten Judenvorstellungen waren eindeutig - ,[...] daß Jud und Preiß oft wie Synonyma für dasselbe Prinzip des Bösen klangen“ (KLEMPERER 2015a:149f.), wusste er ernst zu nehmen. Nach dem Scheitern 
der Räterepublik zeichnete Klemperer auf, dass auch Rote als ,Saujuden“ bezeichnet worden seien (vgl. KLEMPERER 2015a:185). Er betonte zwar, dass er im Kreis der Akademiker nicht unter Antisemitismus gelitten habe, er empfand ihn mehr als Isoliertheit, die für ihn in München bedrückend war und die er mehr als innere Belastung nicht loswerden konnte (vgl. KLEMPERER 2015a:151f.). Ebenso entfremdet fühlte er sich bei der Siegesfeier, bei der er am 10. Mai an der Universität zugegen war. Er befürchtete, als eines der "landfremden Elemente“ betrachtet zu werden, was folgende rhetorische Frage zeigt: „,[W]ürden es diese hier anerkennen, mein Deutschtum? Mußte ich meine Abstammung verbergen? Mir war sehr schwer ums Herz" (KLEMPERER 2015a:186). Den früher von Lydia Rabinowitz an ihn gerichteten Vorwurf: ${ }^{5}$ „Ich habe gehört, daß Sie den Protestanten spielen. Ich verkehre nicht mit konvertierten Juden“ (KLEMPERER 2015a:152) wies Klemperer entschieden bis zur Selbstverleugnung zurück: „Nein, ich spielte ihn nicht. Ich war Protestant, weil ich Deutscher war" (KLEMPERER 2015a:186). Er fühlte sich aufs Tiefste getroffen, als seine Frau Eva während einer der pflichtgemäßen akademischen Besuche von Frau Schick, selbst Engländerin, „fließend Deutsch sprechend, aber mit penetrant englischem Akzent" vertraulich angesprochen wurde: ,,Wir Frauen“ müßten jetzt zusammenhalten, um die Wunden des Krieges zu heilen." Ihrer Meinung nach haben weder Engländer noch Deutsche noch Franzosen, „niemand, nein niemand habe dieses Morden auf dem Gewissen außer ganz allein die Juden, denen allein er Gewinn gebracht habe“ (KLEMPERER 2015a:97). Bei dem nächsten Besuch dieses Sonntags, diesmal bei einem Geschichtsprofessor, sagte Klemperer, er sei zwar mit Eisners Politik nicht einverstanden, aber die Mordtat des Grafen Arco werde noch Verwirrung bringen. Dies verursachte „einen hysterischen Anfall“" der vornehmen Gastgeberin „,Sie wagen es, den Grafen einen Mörder zu nennen', und weiter schrie sie in tiefster Erregung: ,Aber er hat sich für uns geopfert, [...] er hat uns von dem Galizier befreit, ich verehre ihn wie einen Erlöser [...]““ (KLEMPERER 2015a:98). Klemperer nahm auch den Münchener Universitätskreis unter die Lupe. Die Eskalation der antisemitischen Ausbrüche an der Münchener Universität, die gegen eine „radikale Studentengruppe, die mit den neuen Regierungsmännern“ (KLEMPERER 2015a:105f.)

Lydia Rabinowitz war die Schwägerin seines Kollegen an der Münchener Universität, Eugen Lerch. Klemperer bezeichnete sie als „fanatische Zionistin“ (KLEMPERER 2015a:152). Sie habe 1919 einen Artikel im Bayrischen Kurier publiziert, in dem sie den Deutschen immer noch nicht genug Antisemitismus und den Juden, die sich zum Deutschtum bekannten, den Verrat des Zionismus vorgeworfen habe. 
ging, notierte er schon einen Tag nach Ausruf der Räterepublik am 8. April 1919. In den folgenden Tagen mündete die sich ständig verschlechternde Situation in der Stadt in antisemitische Straßendemonstrationen (KLEMPERER 2015a:115f.). Rückschauend, durch spätere Erfahrungen vorbelastet, schrieb Klemperer 1942, die Bürger seien sich dessen nicht bewusst,

daß das republikanische Spiel, dem sie bisher halb apathisch, halb mißmutig zugesehen hatten, für sie doch wohl Schlimmeres bedeuten konnte als nur eine wilde karnevalistische Veranstaltung, wie zeigen sie jetzt ihr Erwachen zum Widerstand? Durch spontanen Antisemitismus. (KLEMPERER 2015a:149)

Von Anfang an war er sich jedoch dessen bewusst, dass die Mehrheit der Professoren und Studierenden schon früher reaktionär und nicht demokratisch gesinnt war (vgl. KLEMPERER 2015a:99, 151). Er hielt sich damals selbst für einen Gegner sowohl der Ideen von Eisner und Landauer als auch für einen Opponenten ,gegen den Antisemitismus der Bürgerschaft und der Studenten“ (KLEMPERER 2015a:189).

In der Schilderung der politischen Wirren der Münchener Republik nahmen Erinnerungen an den Alltag der Münchener Universität einen bedeutenden Platz ein. Klemperer war als Romanist, als Privatdozent für moderne französische Literatur an die Ludwig-Maximilians-Universität gekommen. Nach wie vor war für ihn die Möglichkeit „des Kolleghaltens“ (KLEMPERER 2015a:67) das größte intellektuelle Kapital, das ihn bei seiner wissenschaftlichen Arbeit anregte. Klemperer sollte junge Kriegsheimkehrer unterrichten: „[S]ie waren reifer und verwilderter als normale Studierende“ (KLEMPERER 2015a:67). Die einen besuchten seine Veranstaltungen im ,Kriegsnotsemester' und die anderen zur Vorbereitung auf das Abitur auf dem zweiten Bildungsweg. Sie waren mehr am Praktischen interessiert, deshalb zeigten manche Desinteresse am angebotenen Lehrstoff (vgl. KLEMPERER 2015a:37-39, 68f.). Schon im Mai begann die Anwerbung zum konterrevolutionären Freikorps Epps, in das viele von ihnen eintraten, und das verlorene Semester sollte ihnen dann angerechnet werden (vgl. KLEMPERER 2015a:69, 173, 181, 185-187). Auch Klemperer unterschrieb, ähnlich wie viele andere Akademiker (beispielgebend für die Studentenschaft) die Verpflichtung, der Notreserve beizutreten (KLEMPERER 2015a:187). Im Revolutionstagebuch gibt es viele öffentliche Szenen, ausführliche Schilderungen der politischen Situation und lustige Geschichten, mit denen er wie kaum jemand sonst zu spielen vermag. Schon am Anfang seines Aufenthaltes in München, in der ersten Fakultätssitzung, kommentierte Klemperer mit Ironie die an der Münchener Universität herrschenden Verhältnisse als „eine erfreuliche Aussprache und Fühlungsnahme der Fakultät in schwerer Zeit!“ (KLEMPERER 
2015a:39). Immer wieder, wenn irgendwelche dienstlichen Angelegenheiten von den anwesenden Professoren und Dozenten besprochen wurden - Organisation der „Wiederholungs- und Fortführungskurse“ oder Detailfragen der Entlohnung -, wurden sie ,einer Kommission zur meritorischen Behandlung [...]“ (KLEMPERER 2015a:39) weitergeleitet. Klemperer fragte gleich Professor Vossler, was denn in diesem Fall ,meritorisch' sei. Sein Sitznachbar antwortete ungerührt: „,Ein österreichisches Wort', [...], ,Und was bedeutet es?‘ - ,Gar nichts. ‘ - ,Und was ist der Sprecher? ${ }^{\text {- }}$ - ,Seelenschlosser“"6 ${ }^{\text {(KLEMPERER }}$ 2015a:38f.). Unter den komischen Szenen gibt es auch die Beschreibung einer Dozenten-Wacht, die von dem Philosophen, einem sogenannten „Galimathias Meier" (KLEMPERER 2015a:242) ${ }^{7}$ an der Hintertür der von den Spartakisten befreiten Universität gehalten wurde. Dieser „friedliche[...] Mensch und sehr angenehme[...] Kollege“ (KLEMPERER 2015a:187f.) stand mit einem ungesicherten Gewehr, wovon er keine Ahnung hatte, auf seinem Posten, von dem aus er schon einen unbeabsichtigten Schuss in die Decke abgegeben hatte. Die Lächerlichkeit geht aber im Revolutionstagebuch oft in Bitterkeit über.

In den letzten Tagen der Räterepublik (vgl. KLEMPERER 2015a:153) drehte sich das Karussell der Macht schneller denn je, indem Rote Truppen durch Weiße Truppen in der Stadt ersetzt wurden (vgl. KLEMPERER 2015a:145-154, 161). Klemperer erlebte in München das Ende der Räterepublik. Er beobachtete den blitzschnellen Machtwechsel und das Verhalten der Menschen beim umstandslosen Umtauschen eines roten Bandes gegen ein weißes. Er staunte darüber, wie diejenigen Anhänger, die ,gestern noch ,geschlossen“ hinter der Räterepublik gestanden [sind]“ (KLEMPERER 2015a:160f., 185), ihre politische Ausrichtung änderten. In seinen Erinnerungen nehmen politische SympathieÄußerungen viel Platz ein. Weder den linksradikalen Spartakisten noch den nationalistischen Rechten brachte er Sympathie entgegen (vgl. KLEMPERER 2015a:37). Seine politische Orientierung schien aber noch schwankend zu sein (vgl. WETTE 2015:212). Während der Wahlen zur Nationalversammlung am 19. Januar 1919 entschied er sich für die Liberalen (DDP - Deutsche Demokratische Partei) (vgl. KLEMPERER 2015a:62), obwohl er in früheren Jahren einmal die Sozialdemokraten (SPD) gewählt hatte (vgl. KLEMPERER 2015a:61). Im Grunde genommen stand er auf der Seite der gemäßigten Weimarer Koalition (vgl. KLEMPERER 2015a:187). Privat galten Klemperers Sympathien vor allem

So bezeichnete man Philosophen und Psychologen der philosophisch-naturwissenschaftlichen Fakultät.

Matthias Meier war katholischer Philosophieprofessor in München. 
Studierenden und Akademikern (vgl. KLEMPERER 2015a:69f., 185). Im Chaos nach dem Ersten Weltkrieg setzte sich Klemperer für die Verschränkung von Wissenschaft und Politik ein, „für den Zusammenhalt des Vaterlandes, für Frieden und Ordnung im Innern" (KLEMPERER 2015a:150). Im Revolutionstagebuch brachte Klemperer auch seine größten Antipathien zum Ausdruck: gegen die Revolution (vgl. KLEMPERER 2015a:37), das Münchener Bürgertum mit seinem ,engherzigen Partikularismus“, Apathie und Missmut (KLEMPERER 2015a:149) sowie gegen das Eppsche Korps (KLEMPERER 2015a:37). Er war sich schon damals seiner Rolle als Zeitzeuge bewusst und lernte sogar einige Protagonisten der Münchener Ereignisse im Jahre 1919 kennen. Ausführlich porträtierte er in seinem Revolutionstagebuch Kurt Eisner (vgl. KLEMPERER 2015a:49f., 113f.), Gustav Landauer (vgl. KLEMPERER 2015a:102f., 106, 113, 124f., 128f., 142f., 178) und Max Levien (vgl. KLEMPERER 2015a:102, 142f., 178), aber auch den DDP-Politiker Ludwig Quidde (vgl. KLEMPERER 2015a:109112), Karl Escherich (vgl. KLEMPERER 2015a:53f.), den er mit dessen Bruder Georg Escherich, dem Gründer der ,Organisation Escherich“ (,Orgesch') verwechselte (vgl. WETTE 2015:227) ${ }^{8}$, und den Freikorpsführer Franz Epp (vgl. KLEMPERER 2015a:113f., 178, 187), der mit seinen Truppen die Räterepublik niederschlug. In einer Notiz vom 17. Januar 1920 beschrieb Klemperer eine Szene zum Ausverkauf der Privatbibliothek des ermordeten bayrischen Ministerpräsidenten Kurt Eisner als „Bibliothek eines Journalisten, der mehr Interessen als Geld besessen hat" (KLEMPERER 2015a:191). Obwohl er ihn in einer fast ,folkloristisch' gefärbten politischen Versammlung einen Monat nach dessen Amtseintritt ironisch einschätzte - „dieser Feuilletonist hatte den bayrischen Thron gestürzt und war jetzt Beherrscher Bayerns“ -, äußerte sich Klemperer diesmal lapidar über Eisner (vgl. KLEMPERER 2015a:52).

Als Zeitzeuge machte er auf die fortschreitende Eskalation der Gewalt, Missachtung der Bürgerrechte, Straßenkämpfe, Legitimation der politischen Morde, Geiselmorde und den Tod auf den Münchener Straßen sowie kärgliche Lebensmittelvorräte usw. aufmerksam (vgl. KLEMPERER 2015a:72f.). Vom Februar bis zum Mai 1919 nahm er alles auf und gestand, sich selbst in einem inneren Zwiespalt zu befinden: „[A]lles ist jämmerlich, und alles ist blutig, man möchte immer weinen und lachen in einem“" (KLEMPERER 2015a:192). Flugblätter, Plakate, Maueranschläge, Zeitungsersatzblätter ebenso wie die Modewörter der Revolution (Truppen, Gemeinden, sich geschlossen hinter eine Sache

8 Die „Organisation Escherich“ entstand nach dem Verbot der Einwohnerwehren in Bayern. 
stellen, in einer Sache verankert werden) (vgl. KLEMPERER 2015a:148f.) wurden ihm zu gleichberechtigten Zeichen, aus denen sich zusammen mit den Ereignissen die in ihnen potenziell innewohnende Tragikomik der Münchener Revolution ergab. Der Charakter der Münchener schien ihm zwar rätselhaft, er entlarvte aber ihre Eigenheiten mit zugespitzter Ironie, indem er häufig auch Wort- und Situationskomik berücksichtigte:

Man muß die schöne Gemütsruhe des Münchener Bürgertums miterlebt haben, um den gelungenen Handstreich der Rätepartei nicht allzusehr zu bewundern. Ahnungslos waren die Bürger und alle Gemäßigteren diesmal nicht, das tuschelte überall seit Wochen, im April käme ,es'. Aber der gute Bürger dachte eben, er habe sich lange genug politisch aufgeregt, und einmal müsse der Mensch auch,sei Ruh` haben. Also kümmerte man sich um Butter und Eier statt um Mühsam und Landauer. (KLEMPERER 2015a:109)

Klemperer würzte sein Tagebuch mit vielen humorvollen Anekdoten, Geschichten und Beschreibungen (vgl. KLEMPERER 2015a:35, 131f., 135, 157f., 187f.), was ihn als einen äußerst talentierten Journalisten sowie einen scharfsinnigen Beobachter ausweist. Im Revolutionstagebuch gehen Lächerlichkeit und Bitterkeit ineinander über. Klemperer nannte zwei fremde Erscheinungen in der Räterepublik, in denen tragische und komische Aspekte miteinander verbunden waren (KLEMPERER 2015a:131) - die Rote Armee und das Revolutionstribunal. Jedoch überwiegt im Revolutionstagebuch die tiefergreifende Reflexion des Chronisten Klemperer, der konstatierte: „,[E]s ist tragisch für den uninteressierten Zuschauer. Wie schade, daß man nicht gleichzeitig bloßer Zuschauer sein kann, wenn man Deutscher ist“" (KLEMPERER 2015a:194).

\section{Literatur}

BIEBER, HANS JOACHIM (1991): Bürgertum in der Revolution. Hamburg.

Bischoff, William (1977): ,, The Action Committee of Revolutionary Artists in the Munich Revolution of 1918-19“. In: Studies in Modern European History and Culture 3:7-36.

BuRSCHELL, FriedRICH (1962): Revolution und Neue Erde. München 1918/1919. Aus meinen Erinnerungen. In: Imprimatur Neue Folge 3:244-248.

ChoŁuj, Bożena (1991): Deutsche Schriftsteller im Banne der Novemberrevolution 1918. Bernhard Kellermann, Lion Feuchtwanger, Ernst Toller, Erich Mühsam, Franz Jung. Wiesbaden.

GRAU, BERnHARD: Revolution, 1918/1919. In: Historisches Lexikon Bayerns 09.05.2008 (19.05.2019).

Online verfügbar: https://www.historisches-lexikon-bayerns.de/Lexikon/Autor:Grau, Bernhard 
HafFner, Sebastian (1991): Die deutsche Revolution 1918/19. München.

Höller, RAlF: „Aus ist's! Revolution! Marsch! “. In: Neues Deutschland 29.12.2018 (18.05.2019).

Online verfügbar: https://www.neues-deutschland.de/artikel/1108916.bayerischeraeterepublik-aus-istrs-revolution-marsch.html

JACOBS, Peter (2010): Victor Klemperer. Die Biographie. Berlin.

Kittstein, Ulrich / Zeller, Regine (eds.) (2009): „,Friede, Freiheit, Brot! “ Romane zur deutschen Novemberrevolution. Amsterdamer Beiträge zur neuen Germanistik 71.

KLEMPERER, VICTOR (2015a): Man möchte immer weinen und lachen in einem. Revolutionstagebuch 1919. Berlin.

- (2015b): LTI. Notizbuch eines Philologen. Berlin.

- (2015c): Ich will Zeugnis ablegen bis zum letzten. Tagebücher 1942-1945. Berlin.

Kluge, Ulrich (1985): Die deutsche Revolution 1918/1919. Staat, Politik und Gesellschaft zwischen Weltkrieg und Kapp-Putsch. Frankfurt a. M.

KREILER, KURT (1978): Die Schriftstellerrepublik. Zum Verhältnis von Literatur und Politik in der Münchner Räterepublik. Ein systematisches Kapitel politischer Literaturgeschichte. Berlin.

Mann, Klaus (1967): Kind dieser Zeit. Reinbek bei Hamburg.

MeYer, GeORG (1977): Bibliographie der deutschen Revolution 1918/19. Göttingen.

Müller, Hermann (1929): Die November Revolution Erinnerungen. Berlin.

REICHWEIN, MARC: Wie Victor Klemperer die Münchner Räterepublik sah. In: Die Welt 11.07.2015, (28.08.2017).

Online verfügbar: https://www.welt.de/kultur/literarischewelt/article143850383/WieVictor-Klemperer-die-Muenchner-Raeterepublik-sah.html

Schmalzl, Markus: Zweite Revolution, 1919. In: Historisches Lexikon Bayerns 25.09.2017 (19.05.2019).

Online verfügbar: https://www.historisches-lexikon-bayerns.de/Lexikon/Zweite Revolution,_1919/ (25.09.2017).

Weidermann, Volker (2017): Träumer. Als die Dichter die Macht übernahmen. Köln. WeILler, EdITH (1994): Max Weber und die literarische Moderne. Ambivalente Begegnungen zweier Kulturen. Stuttgart / Weimar.

Wette, Wolfram (2015): Die deutsche Revolution von 1918/19. Ein historischer Essay. In: Man möchte immer weinen und lachen in einem. Revolutionstagebuch 1919. Berlin, 197-216. 